\title{
Performance Analysis of Internet of Things using Visible Light Communication
}

\author{
B R Vatsala, C Vidya Raj
}

\begin{abstract}
Internet of Things enables seamless interaction between connected devices. The growing popularity of IoT will increase the number of sensors and devices to be connected with Internet enormously, resulting in generation of Trillions of GBs of data. Most of the IoT devices have very less storage capacity and hence data generated are to be transmitted to IoT node head which takes care of processing. Data generated from ECG devices, Video surveillance systems are very large requiring a physical medium with high bandwidth for the connection between device and IoT node head. Further the objects plugged into Internet are most of the times powered by batteries requiring low power communication. Visible Light Communication (VLC) is one such technology that provides wide bandwidth up to 10Gbps with energy efficiency and thus it can be a potential solution for the above problem. In this paper we propose NS3 based IoT implementation using existing IoT protocol stack with Visible Light Communication as the physical medium considering error model. We achieve a throughput of 416 Mbps which is a significant improvement over Wireless Fidelity based IoT implementation which has a throughput of 91.2 Mbps under the same condition.
\end{abstract}

Keywords : Internet of Things, NS3 Network Simulator, Visible Light Communication, WiFi

\section{INTRODUCTION}

IoT is the emerging technology to seamlessly connect physical devices to the Internet [1]. Big data are generated by IoT systems [15] such as sensor data by weather monitoring systems, data generated by wearable health care and fitness devices, data generated by vehicle tracking etc. The generation of big data in many IoT systems necessitates the need for a high bandwidth wireless physical medium. VLC is one such wireless medium having bandwidth in terms of Giga hertz [2]. Visible light is harmless for human eyes as a communication medium. VLC is free from security attacks as visible light cannot penetrate through walls. Various wireless devices such as HDTV, Smart phone, Printer desktops can be connected using VLC.

Generation of data from VLC technology is detailed in [3], it uses a part of the electromagnetic spectrum which is unlicensed namely- The Visible Spectrum. Here the on and off flickering of the LEDs represents logic 1 and 0. Different data strings can be generated by changing rate of flickering. The rapid modulation of intensity of LED cannot be noticed by human eyes, hence we observe constant output.

Revised Manuscript Received on December 12, 2019

* Correspondence Author

B R Vatsala*, Department of Computer Science and Engineering, The National Institute of Engineering, Mysuru, India.

Email: vatsalabr@nie.ac.in

Dr C Vidya Raj, Department of Computer Science and Engineering, The National Institute of Engineering, Mysuru, India.

Email: vidyarajc@nie.ac.in

Krishna Kadam et. al. articulates the use of Visible Light technology for IoT in [16], where they propose an architecture combining the benefits of IoT and VLC system.

Chia-Wei Chen et.al. highlights the shortage of radio-frequency spectrum and demonstrate that VLC can be a promising solution for it. They implement IoT using VLC over UART system and analyze the Bit Error Rate with respect to transmission distance in [17].

We propose the implementation of IoT in NS3 using Visible Light as the physical medium. The performance (throughput) of this implementation is analyzed with respect to IoT implementation based on $\mathrm{WiFi}$, considering error model in which packets are dropped according to bit error rate set. The main objective of the work is to demonstrate that VLC medium for IoT meets the communication requirement of high bandwidth than WiFi. An Analytical comparison of VLC with other Wireless technologies is also discussed.

This paper is organized as follows: section 2 deliberates on protocol stack for IoT, Section 3 describes existing VLC simulations, Section 4 elaborates VLC module in NS3 that is added to existing NS3 module Section 5 discusses our proposed model for implementation of IoT in NS3 using VLC as well as WiFi as physical medium Section 6 discusses throughput analysis of our proposed model with respect to WiFi based IoT implementation. Section 7 concludes our IoT implementation techniques.

\section{IoT Network Protocol Stack}

To implement IoT we surveyed its protocol stack. IoT protocol suite contains adaptation layer along with existing TCP/IP layers. The various layers of IoT Network protocol stack are discussed below.

\section{a) Physical and MAC Layer}

The main constraints in most of the IoT applications are low power and wireless physical medium. The IEEE 802.15.4 which is low power, wireless personal area networks is the potential solution for these constraints. It specifies standards and protocols for lower layers namely physical and MAC layers.

The physical layer is based on DSSS technique with a transfer rate ranging from $20 \mathrm{kbps}$ to $250 \mathrm{kbps}$ and operates in frequency band of $868 \mathrm{MHz}, 915 \mathrm{MHz}$ or $2450 \mathrm{MHz}$.

It requires very less power of around one milliwatt but has extremely short range which is about $10 \mathrm{~m}$. Thus it is mainly suitable for indoor environment.

The MAC layer defined in this provides management service along with data service. Medium access mechanism is CSMACA. It also uses energy scan algorithm to transmit frames in channels with low energy. 
The services provided here are framing, flow control, Error Control, Reliable and Robust communication. The frame size is 127 bytes. The frame structures defined are: Beacon Frame, Data Frame, Acknowledgement Frame and MAC Command Frame. J. W. Hui et.al. make an extension for IP over WPAN to enable communication between low power tiny devices that need a long battery life in [4].

\section{b) Adaptation Layer}

IoT uses IPV6 as the communication protocol at the network layer as it has large address space. It has MTU of 1280 bytes but as discussed above MTU of 802.15.4 is only 127 bytes. The adaptation layer is responsible for compatibility between these two protocols.

D. Culler and S. Chakrabarti in [5] discussed about 6LoWPAN which stands for IPv6 over low power wireless personal area networks, for communication of IoT devices using IPv6 over the IEEE 802.15.4 protocol. All IP based devices in the Internet can communicate with 6LoWPAN devices due to the adaptation layer. The operations performed at the adaptation layer are Header compression, Fragmentation and link layer forwarding.

\section{c) Network Layer}

IPV6 is the protocol used for addressing in IoT due to its large address range. The routing protocols of IoT are based on constraints such as avoiding non encrypted links (secure transmission), minimize latency (high speed transmission), paths with low power etc.

Vasseur, et.al have designed ripple routing protocol (RPL) for Low Power and Lossy Networks (LLNs) in [6], RPL is distance vector routing protocol. It constructs destination oriented directed acyclic graph for routing purpose based on distance vectors exchanged and The objective function based on constraints.

\section{d) Transport Layer}

UDP is the good choice for communication in low power environments than TCP as it low weight, connectionless protocol and has less overhead. However in some applications where reliable service is required TCP is preferred.

\section{e) Application layer}

IoT Application layer protocols are chosen based on specific requirements, for example net banking applications require reliable and secure communications, a network where large sensors are connected to a gateway need low power transmission. Vedio surveillance systems need low latency, high bandwidth etc

Constrained Application Protocol (CoAP) is the request response protocol based on UDP that provide web services in resource constrained environment. It takes care of reliable service by sending acknowledgement message for the data message received. W. Colitti,et.al, evaluated CoAP over HTTP in wireless sensor networks [7]. The performance with respect to energy consumption and response time is evaluated and they demonstrate that CoAP is more suitable for REST (Representational State Transfer) based applications with less energy usage and response time.

MQTT (Message Queue Telemetry), is the application protocol that runs in large network of small devices with constraints such as low bandwidth connections, unreliable network limited processing and storage capacity. It is mainly used in remote monitoring systems. It is publish/subscribe protocol using TCP. It was developed by IBM mainly as a client/server protocol. The message format specification for fixed header, variable header, payload, and message identifiers are detailed in [8]. It also gives specifications for command messages and flows.

Other application protocols used extensively in IoT are, XMPP specially designed for chatting services, AMQP which gives reliable service in net banking services

\section{EXISTING VLC Simulations}

The physical medium we are using in our work is Visible Light Communication. Simulations and analysis carried out so far with respect to VLC are referenced as mentioned below.

H. Nguyen et. al. have proposed a Simulation program for indoor visible light communication system in MATLAB and Simulink [9]. The simulation program is used to analyze received signal waveform, RMS delay and Illumination distribution.

F. Miramirkhani et. al. Have investigated channel models for visible light communication using Zemax[10] to obtain channel response in various indoor settings. They studied the effect of multi transmitter deployment, position / rotation of transmitter / receiver, desk light, surface materials and furniture on RMS delay spread and DC gain of the channel

B. Tomas has developed high performance VLC physical medium in JIST [11]. He modified MAC layer protocol in accordance with VLC and calculated receiver power for VLC path loss and found that VLC does experience specific path loss with respect to angle of entrance and exit of light at directed antennas.

Adel Aldalbahi et. al. have developed NS3 based open source module for VLC [12]. They developed test bed implementation of VLC using GNURadio and validated their new module implementation of VLC in NS3 by setting VLC point to point link between two nodes and analyzing the simulation result of the link in terms of SNR, Goodput, Error rate of symbol and packet with that of test bed implementation. They set up two different scenarios, in scenario 1 transmitter is placed directly above the receiver and varied the distance, in scenario 2 receiver is tilted horizontally and varied angle of acceptance. In both cases they demonstrated that there is considerable match in test bed and simulation implementation of VLC

NS3 is the open source platform for simulation of Internet Protocols and for real time modeling of network applications. It is based on object oriented Programming model written in $\mathrm{C}++[13]$. New modules can be integrated to NS3 providing scope for extension. It is apt for research and development. Hence we chose NS3 as the best module to implement IoT over VLC medium 


\section{VLC MODULE IN NS3}

Visible Light Communication module developed by authors in [12], contains classes which are inherited using point-to-point module in NS3. The primary modules consist of the following:

(i) VLC net device: It has variables and methods do define VLC network device parameters: address, position, azimuth and height.

(ii) VLC TX net device: It contains additional attributes for transmitter with respect to transmitted signal power, transmitter gain and radiance angle.

(iii) VLC RX net device: It also contains additional attributes for receiver with respect to photo detector area, FOV angle, filter gain concentration gain etc.

(iv) VLC Mobility Model: It has attributes for capturing movement the device such as transmitter and receiver location, rotation; it provides call back functions to capture state values whenever the device changes its position.

(v) VLC Error Model: implements packet drop error model according to distribution of bit error rate. Modulation schemes such as PSK, QAM and VPPM are available here.

(vi) VLC SNR Model: This class has functions to compute signal to noise ratio along with optical signal power.

(vii) VLC channel model: It has methods to create channel between transmitter and receiver. It consists of variables to set channel parameters such as temperature, wavelength, noise signal etc.

(viii) VLC Helpers: It contains methods to link vlc channel between vlc net devices and to set channel attributes etc.

\section{IMPLEMENTATION OF IOT IN NS3}

IoT implementation consists of two node objects with 6LoWPAN installed on them. Internet stack is also installed on them with IPV6 addressing scheme.

In scenario1, the nodes are linked using VLC channel attached with VLC Transmitter (T) and Receiver (R) devices as shown in Fig 1. The Transmitter is set with attributes such as semi Angle, Azimuth, Elevation, gain and data rate. The Receiver is set with attributes such as Filter Gain, Refractive Index, FOV Angle, Concentration Gain, RX Gain and Photo Detector Area. The receiver is also set with VlcErrorModel:PSK

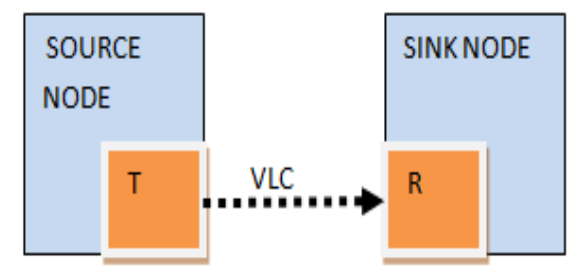

Fig 1: IoT nodes with VLC link

In scenario2, the nodes are linked using WiFi channel with WiFi physical standard 80211b as shown in Fig 2, physical mode is set to Dsss Rate 1Mbps and error model used is YansErrorRateModel.

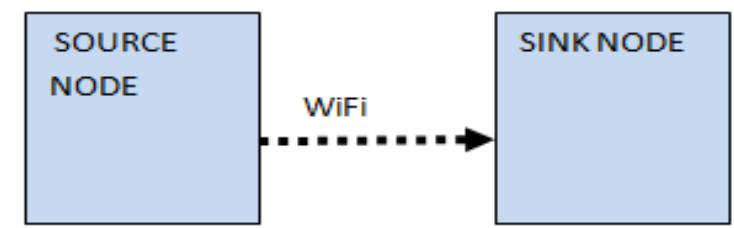

Fig 2: IoT nodes with WiFi link

The Simulation is run for 100 seconds with source sending packets of 1040 bytes at $1 \mathrm{Mbps}$ rate using on-off application which is based on TCP. The acknowledgements from sink are 40 bytes . table 1 consists of parameter values considered for V1C and WiFi implementation of IoT. The channel data rate of VLC and WiFi is in accordance with [14].

Table- I: Simulation Parameters Considered

\begin{tabular}{|l|l|l|}
\hline \multicolumn{1}{|c|}{ Parameter } & \multicolumn{1}{|c|}{ VLC } & \multicolumn{1}{|c|}{ WiFi } \\
\hline Data rate & $1 \mathrm{Mbps}$ & $1 \mathrm{Mbps}$ \\
\hline $\begin{array}{l}\text { Channel } \\
\text { data-rate }\end{array}$ & $1 \mathrm{Gbps}$ & $1 \mathrm{Mbps}$ \\
\hline Rx gain & 10 & 10 \\
\hline Packet Size & 1040 bytes & 1040 bytes \\
\hline Error model & VlcErrorModel:PSK & $\begin{array}{l}\text { ns3::YansErrorRate } \\
\text { Model }\end{array}$ \\
\hline
\end{tabular}

\section{RESULT AND DISCUSSION}

Throughput (bytes received at sink node every one second) analysis of IoT implementation using VLC is considered and it is compared with that of $\mathrm{WiF}$ as in Fig 3. It is obvious from the result that VLC has better throughput than $\mathrm{WiFi}$, since VLC is free from radio interference and has a transfer rate of Giga hertz. We observed throughput of $416 \mathrm{Mbps}$ for VLC based implementation and 91.2 Mbps for WiFi based implementation

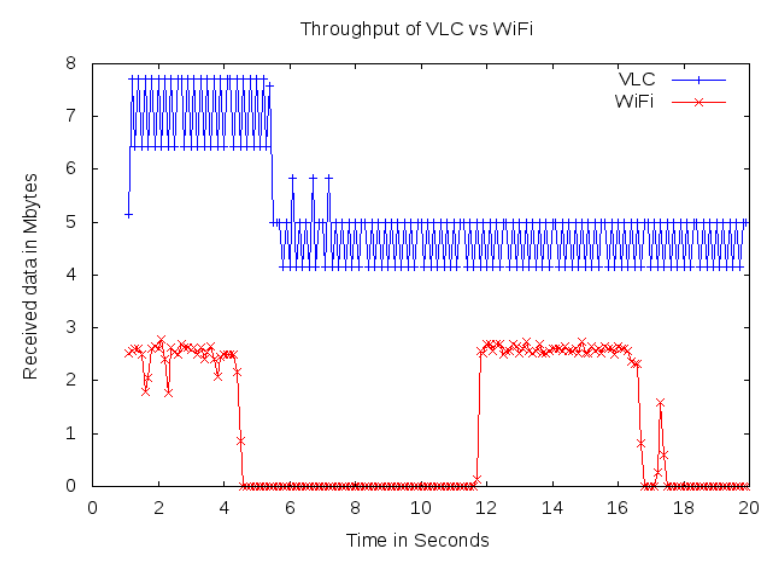

Fig 3: Throughput Analysis of VLC vs WiFi 
Other IoT wireless technologies such as zigbee, Blue tooth, IrDA and LPWAN have data rates less than 1 Mbps hence they are not suitable for IoT applications that require high bandwidth

Visible Light is not harmful to human eyes, it overcomes the radiation related health issues which are present in $\mathrm{WiFi}$. Since visible light cannot penetrate through walls, it is secure. Smart hospitals, Smart homes, military control rooms, aircrafts, museums etc are best places to deploy it.

The main disadvantage of visible light communication is its short range and can be implemented in indoor environment only. VLC is unidirectional hence the communication devices are to be aligned in line of sight condition. It may also face interference problem with sunlight.

The real time implementation of the work is the major challenge as VLC is still under experimental stage. Other IoT parameters such as Battery Life, QoS, Scalability, cost and Payload Length etc are still the areas to be explored

\section{CONCLUSION}

Internet of Things is implemented over visible light communication medium and WiFi medium in NS3. The throughput analysis demonstrates that VLC which has Throughput of $416 \mathrm{Mbps}$ is better when compared to $\mathrm{WiFi}$ that has 91.2 Mbps throughput Visible light is a green medium as it is not harmful and less liable for security attacks. Hence to meet the energy and high bandwidth requirement of IoT, VLC is the best choice.

\section{ACKNOWLEDGMENT}

We express our gratitude to the National Institute of Engineering for providing opportunity to complete this work. We are also grateful to the authors whose papers have been referred.

\section{REFERENCES}

1. L. Atzori, A. Iera, and G. Morabito, "The Internet of things: a survey," Computer Networks, vol. 54, no. 15, pp. 2787-2805, 2010.

2. Haas, Herald "Wireless data from every light bulb,"TED Global. Edinburgh, Scotland, Jul 2011

3. P. H. Pathak, X. Feng, P. Hu, and P. Mohapatra, "Visible light communication, networking, and sensing: a survey, potential and challenges," IEEE Communications Surveys \& Tutorials, vol. 17, no. 4, pp. 2047-2077, 2015.

4. J. W. Hui and D. E. Culler, "Extending IP to low-power, wireless personal area networks," IEEE Internet Computing, vol. 12, no. 4, pp. 37-45, 2008.

5. D. Culler and S. Chakrabarti, "6lowpan: incorporating IEEE 802.15. 4 into the IP architecture, IPSO Alliance," White Paper, 2009.

6. J. Vasseur, N. Agarwal, J. Hui, Z. Shelby, P. Bertrand, and C. Chauvenet, "Rpl: the ip routing protocol designed for low power and lossy networks," Internet Protocol for Smart Objects (IPSO) Alliance 36, 2011.

7. W. Colitti, K. Steenhaut, N. De Caro, B. Buta, and V. Dobrota, "Evaluation of constrained application protocol for wireless sensor networks," Proceedings of the 18th IEEE Workshop on Local and Metropolitan Area Networks (LANMAN '11), pp. 1-6, IEEE, Chapel Hill, NC, USA, October 2011.

8. D. Locke, "MQ telemetry transport (MQTT) v3. 1 protocol specification," IBM developerWorks Technical Library, 2010.

9. H. Q. Nguyen et al., "A MATLAB-based simulation program for indoor visible light communication system,', in Proc. 7th Int. Symp. Commun.Syst. Netw. Digit. Signal Process. (CSNDSP), pp. 537-541, Jul. 2010.

10. F. Miramirkhani, M. Uysal, and E. Panayirci, "Novel channel modelsfor visible light communications,', Proc. SPIE, vol. 9387, p. 93870Q,Feb. 2015 .
11. B. Tomaš, "Visible light communication physical layer design for jist simulation," Res. Papers Faculty Mater. Sci. Technol. Slovak Univ. Technol.,vol. 22, no. 341, pp. 41-46, 2014.

12. Adel Aldalbahi, Michael Rahaim, Abdallah Khreishah , Moussa Ayyash And Thomas D. C. Little, "Visible Light Communication Module: An Open Source Extension to the ns3 Network Simulator With Real System Validation," IEEE Access vol. 5, pp 22144 -22158, october 2017.

13. https://www.nsnam.org/

14. http://www.rfwirelss-world.com

15. Mouzhi Ge , Hind Bangui , Barbora Buhnova ,"Big Data for Internet of Things: A Survey," Elsevier Future Generation Computer Systems, Article in Press, 2018.

16. Krishna Kadam, Manisha R. Dhage ,"Visible Light Communication for IoT," 2nd International Conference on Applied and Theoretical Computing and Communication Technology (iCATccT), pp 275-278,2016.

17. Chia-Wei Chen, Wei-Chung Wang et. Al "Visible light communications for the implementation of internet-of-things," Optical Engineering, vol $55,2016$.

\section{AUTHORS PROFILE}

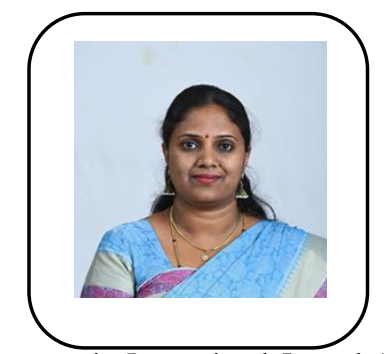

B R Vatsala Assistant Professor, Department of Computer Science and Engineering, The National Institute of Engineering, Mysuru, Karnataka, India. Teaching and Research Interest areas are Internet of Things, Computer Architecture, Compiler Design, Microprocessors, Embedded Systems and Computer Networks. Presented papers in Conferences and published papers in International JournalsAwarded with Elite Certificate with gold medal printed on it for scoring $90 \%$ in NPTEL online Course on "Introduction to Internet of Things" . Got best project award for guiding project titled "Feature Extractor for a Smart Phone to Extract Features from Images of Faces and Currency Notes for the purpose of Outsourced Pattern Matching"

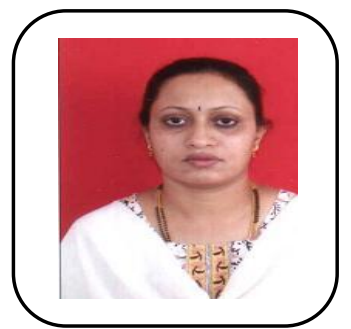

Dr C Vidya Raj Professor, Department of Computer Science and Engineering, The National Institute of Engineering, Mysuru, Karnataka, India. Research and Teaching Interest areas are Quantum Computing, Software Engineering Computer Networks, Wireless networks, Storage Area Networks and Internet of Things. Presented papers in various Conferences and, published papers in reputed International journals. She is life member of Indian society for Technical Education, Fellow member of Institute of Engineers and member of IEEE. Received best paper awards for presenting papers. She is Guiding research scholars. Two research scholars were awarded Doctorate under her Guidance .She has given several technical talks in various institutions 